\title{
NONNEGATIVE IDEMPOTENT MATRICES
}

\author{
RALPH DeMARR
}

ABSTRACT. Elementary ideas in the the ory of partially ordered linear algebras are used to describe the structure of nonnegative idempotent matrices. In particular, we obtain a kind of "spectral decomposition" theorem for such matrices.

Flor has given a description of nonnegative idempotent matrices; see Theorem 2 of [2] or see Plemmons and Cline [3]. Flor's theorem involves permuting rows and columns and therefore cannot in general be applied to infinite matrices. We give another description of nonnegative idempotent matrices based on elementary ideas in the the ory of partially ordered linear algebras. These results can be applied to finite or infinite matrices.

The author wishes to thank Art Steger for his many valuable comments.

A pola (denoted by $A$ ) is a real linear associative algebra which is partially ordered so that it is a partially ordered linear space and $0 \leq x y$ when. ever $x, y \in A, 0 \leq x, 0 \leq y$. We also assume that $A$ has a multiplicative identity $1 \geq 0$. A pola $A$ is said to have the Archimedean property if the following holds: if $x, y \in A$ and $n x \leq y$ for every positive integer $n$, then $x \leq 0$. More details can be found in Birkhoff [1].

Example. For a fixed positive integer $m$ let $A$ be the real linear algebra of all matrices of order $m$ with real entries. We shall partially order $A$ entry by entry so that $A$ becomes a pola which has the Archimedean property. Note that if $d \in A$ and $0 \leq d \leq 1$, then $d$ must be a diagonal matrix. For any positive integer $k$ such that $1 \leq k \leq m$ we define $p_{k}=\left[\delta_{i k} \delta_{k j}\right]$. As usual, $\delta_{i j}$ is the Kronecker delta. Thus, $0 \leq p_{k} \leq 1$ for all $k$ and $p_{1}+\cdots+$ $p_{m}=1$. In particular, note that if $y \in A$ and $p_{k} y p_{k}=0 \in A$, then the $k$ th entry on the main diagonal of $y$ is zero. Again note that if $u=\left[a_{i j}\right] \in A$ and $a_{k k} \neq 0$ for some $k$, then $w=\left(\alpha_{k k}\right)^{-1} u p_{k}$ is an idempotent matrix. In addition, if $u \geq 0$, then $0 \leq w \leq\left(\alpha_{k k}\right)^{-1} u$. Also if $u^{2}=u$, then $u w=w$. It is im-

Presented to the Society, January 16, 1974; received by the editors June 21, 1973 and, in revised form, September 25, 1973.

AMS (MOS) subject classifications (1970). Primary 06A70; Secondary 15A45.

Key words and phrases. Partially ordered linear algebra, matrix inequalities, idempotent matrices. 
portant to note that if $x \in A$, then every column in the matrix $w x$ is a real multiple of some fixed column of $w x$.

Although we will apply our general results only to the example given above, it will be clear that they extend to certain algebras of inf inite matrices.

Lemma 1. Let $A$ be a pola which has the Archimedean property. If a, $u \in A$ are elements such that $0 \leq a \leq 1,0 \leq u=u^{2}$ and aua $=0$, then uau $=0$.

Proof. If we put $h=u a u$, then $u b=h u=h$ and $h^{2}=0$. Since $0 \leq h \leq$ $u$, we get $0 \leq(u-h)^{n}=u-n h$ for every positive integer $n$. Using the Archimedean property, we get $h \leq 0$. Hence, $u a u=h=0$.

We now apply this lemma to our example above. Suppose $a=p_{k}$ and $p_{k} u p_{k}=0$ for some $k$; this means that the kth (main) diagonal entry of $u$ is zero. Since $0=u p_{k} u=\left(u p_{k}\right)\left(p_{k} u\right)$, we can conclude that the $k$ th row of $u$ has all zero entries or the $k$ th column of $u$ has all zero entries.

This lemma may again be applied as follows. Suppose every diagonal entry of $u$ is zero; this means that $p_{k} u p_{k}=0$ for every $k=1, \cdots, m$. Hence, $u p_{k} u=0$ for every $k$. Therefore, $u=u\left(p_{1}+\cdots+p_{m}\right) u=0$.

Lemma 2. Assume the condition of Lemma 1. If $b=1-a$, then $0 \leq$ $b u b=(b u b)^{2}$.

Proof. It is clear that $0 \leq b \leq 1$. Hence, $0 \leq b u b$. Since $u a u=0$, we get $0 \leq u a^{2} u \leq u a u=0$. Similarly we can show that $u a b u=u b a u=0$. Hence, $u=u(a+b)^{2} u=u b^{2} u$ so that $b u b=(b u b)(b u b)$.

We may apply Lemma 2 as follows. Suppose $a=p_{k}$ and $p_{k} u p_{k}=0$ for some $k$; this means that the $k$ th diagonal entry of $u$ is zero. Thus, $\left(1-p_{k}\right)$ - $u\left(1-p_{k}\right)$ is a nonnegative idempotent matrix in which both the $k$ th row and $k$ th column have all zero entries. We may apply this idea again to any zero entry on the main diagonal of $\left(1-p_{k}\right) u\left(1-p_{k}\right)$ and proceed in this fashion until we can write $u=z+v$, where $v \geq 0$, all diagonal entries of $v$ are zero, $0 \leq z=z^{2}$ and $z$ has the following property: if the $k$ th diagonal entry of $z$ is zero, then both the kth row and $k$ th column of $z$ have all zero entries. This is related to Flor's result but does not involve permuting rows and columns. Since $z+v=(z+v)^{2}$, we have $v=z v+v z+v^{2}$. Hence, $0=$ $z v z+z v^{2}=z v z+v^{2} z$. Thus, $0=z v z=z v^{2}=v^{2} z$. It follows that $v^{3}=$ $v z v^{2}+v^{2} z v+v^{4}=v^{4}$. Since $0 \leq v^{3} \leq v$, it is clear that every diagonal entry of $v^{3}$ is zero. Since $v^{3}=v^{4}$, it follows that $v^{3}=\left(v^{3}\right)^{2}$ and we may now use the second application of Lemma 1 to show that $v^{3}=0$.

If we now assume that $u$ is a stochastic matrix with each row sum equal 
to one, then $u$ has the following property: if $x \in A, x \geq 0$ and $x u=0$, then $x=0$. Now $v^{2} u=v^{2} z+v^{3}=0$, so that in this case $v^{2}=0$. Similarly we can show that $z v u=0$, so that $z v=0$.

If we next assume that $u$ is a doubly stochastic matrix, then we can use the above ideas to show that $v=0$. Thus, each diagonal entry of $u$ must be positive because $u=z$.

Lemma 3. Let $A$ be a pola which has the Archimedean property. Suppose $u, w \in A$ are elements such that $0 \leq u=u^{2}$ and $0 \leq w=w^{2}=u w$. If there exists a real $\beta \geq 1$ such that $w \leq \beta u$, then $w u \leq u$.

Proof. It is clear that $w u \leq \beta u$. Let $\lambda \geq 1$ be the smallest real number such that $w u \leq \lambda u$. The Archimedean property must be used here. Hence, $0 \leq(\lambda u-w u)^{2}=\lambda^{2} u-2 \lambda w u+w u$, so that $w u \leq \lambda^{2}(2 \lambda-1)^{-1} u$. It is easily verified that if $\lambda>1$, then $\lambda^{2}(2 \lambda-1)^{-1}<\lambda$. Hence, we must have $\lambda=1$. Therefore, $w u \leq u$.

We now apply Lemma 3 to obtain a kind of "spectral decomposition" theorem for nonnegative idempotent matrices.

Theorem 4. Let $A$ be the pola of matrices described in the example above. Recall that $A$ has the Archimedean property. If $u \in A$ and $0 \leq u=$ $u^{2}$, then $u=z_{1}+\cdots+z_{m}$, where $z_{k} \geq 0, z_{k} z_{n}=\delta_{k n} z_{k}$ and for each $k$ the matrix $z_{k}$ has the following property: every column of $z_{k}$ is a real multiple of the kth column of $z_{k}$.

Proof. Assume that $u=\left[\alpha_{i j}\right]$. If $\alpha_{11}=0$, then define $z_{1}=0$ and then put $u=z_{1}+u_{2}$. Note that in this case $z_{1} u_{2}=u_{2} z_{1}=0, u_{2}^{2}=u_{2}$ and the first diagonal entry of the matrix $u_{2}$ is zero. If $\alpha_{11}>0$, then define $w_{1}=$ $\left(a_{11}\right)^{-1} u p_{1}$. Thus, $0 \leq w_{1}=w_{1}^{2}$ and $u w_{1}=w_{1} \leq\left(a_{11}\right)^{-1} u$. By Lemma 3 we have $w_{1} u \leq u$. Define $z_{1}=w_{1} u \geq 0$ and $u_{2}=u-z_{1} \geq 0$. It is easily verified that $z_{1}=z_{1}^{2}, u_{2}=u_{2}^{2}$ and $z_{1} u_{2}=u_{2} z_{1}=0$. Note that every column of $z_{1}$ is a real multiple of its first column. A lso note that the first diagonal entry of $u_{2}$ is zero because $z_{1} u_{2}=0$.

We next apply the above procedure to the matrix $u_{2}$ but we look at its second diagonal entry. In any case we see as above that we can write $u_{2}=$ $z_{2}+u_{3}$, where $0 \leq z_{2}=z_{2}^{2}, 0 \leq u_{3}=u_{3}^{2}, z_{2} u_{3}=u_{3} z_{2}=0$. Note that every column of $z_{2}$ is a real multiple of its second column. Also note that the first two diagonal entries of $u_{3}$ are zero.

We may proceed in this fashion until we can write $u=z_{1}+\cdots+z_{m}+u_{m+1}$. Since all diagonal entries of $u_{m+1}$ are zero and $0 \leq u_{m+1}=u_{m+1}^{2}$, we can 
use the second application of Lemma 1 to show that $u_{m+1}=0$. It is clear that the matrices $z_{k}$ have the required properties of the theorem.

We now consider the special case where $u$ is a stochastic matrix with each row sum equal to one. This means that $u$ has the property: if $x \in A$, $x \geq 0$ and $x u=0$, then $x=0$. Now for each $k$ we may write $u=z_{k}+v_{k}$. Let $c_{k}$ be the diagonal matrix having a 1 as the ith diagonal entry if $z_{k}$ has a positive entry in its ith row. Otherwise every entry in $c_{k}$ is zero. Note that $0 \leq c_{k}=c_{k}^{2} \leq 1$ and that the following property holds: if $x \in A, x \geq 0$ and $x z_{k}=0$, then $x c_{k}=0$. We put $d_{k}=1-c_{k}$. Note that $c_{k} z_{k}=z_{k}$ and $d_{k} z_{k}=0$.

Since $v_{k} \geq 0$ and $v_{k} z_{k}=0$, we get $v_{k} c_{k}=0$. Also

$$
z_{k} d_{k} u=z_{k} d_{k} z_{k}+z_{k} d_{k} v_{k} \leq 0+z_{k} v_{k}=0 \text {. }
$$

Hence, $z_{k} d_{k}=0$ so that $z_{k} c_{k}=z_{k}$. It follows that $z_{k}=u c_{k}=c_{k} u c_{k}$.

From the above it follows that if $k \neq n$, then $0 \leq z_{n} \leq v_{k}$, so that $z_{n} \leq u d_{k}$ (using the fact that $v_{k} d_{k}=v_{k}$ ). This means that if $k \neq n$, then the following holds: if the $j$ th column of the matrix $z_{k}$ has a positive entry, then all entries in the $j$ th column of the matrix $z_{n}$ are zero. To prove this, use the fact that $z_{k}=u c_{k}$, which means that the jth diagonal entry of $c_{k}$ must be the number 1 . Since $d_{k}=1-c_{k}$, it follows that the matrix $u d_{k}$ has all zero entries in its $j$ th column. The proof is completed by using the inequality $0 \leq z_{n} \leq u d_{k}$.

The reader should note that in the above discussion we only use the fact that $u$ has at least one positive entry in each row. In particular, if $u$ has one column with all positive entries, then every column of $u$ is a real multiple of this positive column.

If we next assume that $u$ is doubly stochastic, then the above ideas can be used to show that $z_{k}=c_{k} u$ and $v_{k}=d_{k} u$. Thus, if $k \neq n$, then $z_{n} \leq d_{k} u$. This means that if $k \neq n$, then the following holds: if the ith row of $z_{k}$ has a positive entry, then all entries in the ith row of $z_{n}$ are zero.

\section{REFERENCES}

1. G. Birkhoff, Lattice theory, 3rd ed., Amer. Math. Soc. Colloq. Publ., vol. 25, Amer. Math. Soc., Providence, R. I., 1967. MR 37 \#2638.

2. P. Flor, On groups of nonnegative matrices, Compositio Math. 21 (1969), 376-382. MR $41 \# 1769$.

3. R. J. Plemmons and R. E. Cline, The generalized inverse of a nonnegative matrix, Proc. Amer. Math. Soc. 31 (1972), 46-50. MR $44 \# 2759$.

DEPARTMENT OF MATHEMATICS, UNIVERSITY OF NEW MEXICO, ALBUQUERQUE, NEW MEXICO 87131 